\title{
Association between lunar phase and sleep characteristics
}

Csilla Zita Turányi ${ }^{1}$, Katalin Zsuzsanna Rónai ${ }^{1}$, Rezső Zoller $^{1,3}$, Orsolya Véber ${ }^{1}$, Mária Eszter Czira $^{1,7}$, Ákos Újszászi ${ }^{1}$, Gergely László ${ }^{1}$, András Szentkirályi ${ }^{1,7}$, Andrea Dunai ${ }^{1,3}$, Anett Lindner ${ }^{1}$, Julianna Luca Szőcs ${ }^{1}$, Ádám Becze ${ }^{1}$, Andrea Kelemen ${ }^{1}$, Zsófia Lendvai ${ }^{1}$, Miklos Z Molnar ${ }^{5,6}$, István Mucsi $^{1,2,6}$ and Márta Novák ${ }^{1,4}$

${ }^{1}$ Institute of Behavioral Sciences, Semmelweis University, Budapest, Hungary

${ }^{2}$ Institute of Pathophysiology, Semmelweis University, Budapest, Hungary

${ }^{3} 1$ st Dept. of Internal Medicine, Semmelweis University, Budapest, Hungary

${ }^{4}$ Dept. of Psychiatry, University Health Network, University of Toronto, Toronto, Canada

${ }^{5}$ Harold Simmons Center for Kidney Disease Research and Epidemiology, Division of Nephrology and Hypertension, University of California Irvine Medical Center, Orange, CA; USA

${ }^{6}$ Dept. of Medicine, Division of Nephrology, University Health Network, University of Toronto, Toronto, Ontario, Canada

${ }^{7}$ Institute of Epidemiology and Social Medicine, University of Muenster, Germany

CORRESPONDING AUTHOR: István Mucsi, MD, PhD, Division of Nephrology, University of Toronto, Toronto General Hospital, University Health Network, 585 University Avenue, Peter Munk Building, 11c-1268, Toronto ON M5G 2N2

Tel.: 416-340-4084

Phone: (416) 340-4084 
Fax: (647) 689-3070

e-mail: istvan@nefros.net

\section{WORD COUNT}

Abstract:

230

Main text:

2938

Tables:

4 


\begin{abstract}
Objectives: Popular belief holds that the lunar cycle affects human physiology, behavior and health, including sleep. To date, only a few and conflicting analyses have been published about the association between lunar phases and sleep. Our aim was to analyze the relationship between lunar phases and sleep characteristics.
\end{abstract}

Methods: In this retrospective, cross-sectional analysis data from 319 patients who had been referred for sleep study were included. Individuals with apnea-hypopnea index $\geq 15 / \mathrm{h}$ were excluded. Sociodemographic parameters were recorded. All participants underwent one-night standard polysomnography. Associations between lunar cycle (new moon, full moon and alternate moon) and sleep parameters were examined in unadjusted and adjusted models.

Results: $57 \%$ of patients were males. Mean age for men was $45 \pm 14$ years and $51 \pm 12$ years for women. 224 persons had their sleep study done during alternate moon, 47 during full moon and 48 during new moon. There were no significant differences regarding age and gender between groups. Full moon was associated with lower sleep efficiency (median(\%)[IQR] new moon 82[18], full moon 74[19], alternate moon 82[15], $\mathrm{p}<0.001)$, less deep sleep (median(\%)[IQR] new moon 9[9], full moon 6[4], alternate moon 11[9], $\mathrm{p}<0.001$ ) and increased REM latency (median (minutes)[IQR] new moon 98[74], full moon 137[152], alternate moon 97[76], $\mathrm{p}<0.001$ ), even after adjustment for several covariables.

Conclusion: Our results are consistent with a recent report and the widely held belief that sleep characteristics may be associated with the full moon.

Key words: sleep parameters, lunar cycle, full moon, polysomnography 


\section{INTRODUCTION}

The human culture has been very much interested in the cycles of the moon [1]. Beliefs in lunar effects on sleep date back to ancient times. The passage from full to crescent moon provided the basis for the earliest calendars of most civilizations and can be linked to Assyrian and Babylonian mythology. Nevertheless, evidence for the supposed influence of the moon on human behavior has proven particularly elusive. Surveys of workers in the mental health professions, however, show a persistent belief that the full moon may influence behavior [2, 3].

The observations made by Galileo Galilei of the phases of the moon cycle remain correct in today's astronomical understanding. There are 8 phases of the moon cycle, which succeed each other with a periodicity of 3.69 days: new, waxing crescent, first quarter, waxing gibbous, full, waning gibbous, last quarter, waning crescent. The synodic cycle is the interval between 2 successive new moons. This is the time it takes the moon to complete an orbit around the earth (29.53 days), corrected for the earth's orbit around the sun. Most studies associated with the influence of the moon on human behavior focus mainly on the full moon within the synodic cycle [4].

Several published reports suggest an association of the lunar cycle with menstrual cycle $[5,6]$. The lunar cycle also has been suggested to have an impact on fertility and births [7-9]. However, no significant effect of the lunar cycle on the number of deliveries was found in Austria [10] and in American studies [11, 12]. Several papers reported associations between the lunar cycle and the frequency of hospital admissions due to various causes: cardiovascular disease [13], acute coronary events [14], gastrointestinal hemorrhage [15] and infectious diarrhea [16]. Lunar phases were also associated with the number of requests for appointment at a thyroid outpatient clinic [17]. On the other hand, a number of studies showed insufficient evidence or no association between various health-related events and the synodic lunar cycle [18-24]. Studies also showed that lunar periodicity 
may be associated with traffic accidents [25], crime[26], suicides, aggravated assaults and psychiatric emergency room visits [7]. Little is known, however, about the association between the lunar cycle and sleep patterns.

Röösli et al. studied 31 volunteers and showed an association between lunar cycle and sleep characteristics. Subjective sleep duration was shortened and fatigue was increased at full moon. In contrast, sleep latency, wake up events, level of distress, mood and well-being were not associated with the lunar cycle [27]. A study monitoring wrist-activity showed no association of sleep duration and moon phase [28]. Lastly, the moon cycle had no effect on human daily rhythm, wake-up and tosleep times in 4 volunteers [29]. An analysis of diaries kept by 196 participants showed no association between full moon and dream recall [30]. However, a recent study including 33 participants found that electroencephalogram delta activity during NREM sleep, an indicator of deep sleep, decreased by $30 \%$, time to falling asleep increased by $5 \mathrm{~min}$, and EEG-assessed total sleep duration was reduced by $20 \mathrm{~min}$ around full moon [31].

In the present work, we analyzed the relationship of synodic lunar phases with subjective and objective sleep characteristics. Specifically, we wanted to test the hypothesis that full moon is associated with lower sleep efficiency and deep sleep. We also wanted to quantify the association of full moon and sleep latency. There is a strong link between gender and sleep both in the structure of sleep as in the incidence of various sleep disorders $[32,33]$. Accordingly, we have analyzed if the association between the lunar cycle and sleep was different in the sexes.

\section{METHODS}

\section{Sample of patients and data collection}


This retrospective, cross-sectional study was performed at the 1st Department of Internal Medicine, Semmelweis University, Budapest, Hungary. Data obtained from 319 consecutive patients, referred to the sleep laboratory for sleep studies, were analyzed between between January 2007 and November 2009. Exclusion criteria included technical limitations, moderate and severe obstructive sleep apnea (AHI $\geq 15$ ) and sleep efficiency under $20 \%$.

The ethics committee of the Semmelweis University had approved the study Before enrollment, patients received detailed written and verbal information regarding the aims and protocol of the study and signed informed consent. Socio-demographic information (age, gender, level of education) and details of medical history were collected at enrollment.

\section{Questionnaires}

Participants completed a battery of validated questionnaires, including the Athens Insomnia Scale (AIS) (score range 0-24, with higher scores indicating worse sleep, a cut-off score of 10 was used to identify patients with clinically relevant insomnia [34]) and Center for Epidemiologic StudiesDepression (CES-D) Scale [35] (scores range from 0 -60, with high scores indicating greater depressive symptoms, a cut-off score of 16 was used to estimate the frequency of potentially relevant depression in patients), Stanford Sleepiness Scale (SSS) [36] and subjective sleep log [37]. We also tabulated the Charlson Comorbidity Index [38].

\section{Polysomnography}

Standard, attended overnight polysomnography was performed in four acustically isolated and video monitored units in our sleep laboratory (SOMNOscreen ${ }^{\mathrm{TM}}$ PSG Tele, SOMNOmedics GmBH, Germany, CE0494). Tests were always done on weekdays, "lights off' and "lights on" times were uniform and were set at $9 \mathrm{pm}$ and 6 am, respectively. Sleep architecture was characterized by the 
following parameters: sleep efficiency is the ratio of time spent asleep over the time spent in bed; sleep onset latency: time from lights out until sleep onset (defined as first epoch of stage 2); number of awakenings; arousal index: number of arousals per hour. The amount of four different stages of non-rapid eye movement sleep and REM sleep were determined as percentages of sleep period time (time from sleep onset until final awakening). Light sleep is the sum of stage 1 and 2, slow wave sleep or deep sleep is the combination of stage 3 and 4 . Wake percentage is defined as the percentage of wake period from lights out to lights on. All recordings were scored visually per $30 \mathrm{~s}$ epochs by experienced scorers according to the criteria of Rechtschaffen \& Kales [39].

\section{Definition of sleep disorders}

Apnea was defined as the absence of airflow for more than $10 \mathrm{~s}$, hypopnea was defined as a clearly discernible reduction in airflow for more than $10 \mathrm{~s}$, associated with an arousal and/or reduction in oxygen saturation greater than 3\% [40]. OSA severity was defined by apnoe-hypopnea index (AHI), which is calculated as the number of apnea and hypopnea episodes per hour of sleep.

PLMS was defined by limb movement (LM) duration: $0.5-5 \mathrm{~s}$ with inter-movement interval: 5-90 s. A PLMS cycle consisted of at least four consecutive LMs. The periodic limb movement index (PLMI) was defined as the number of LMs per hours during sleep. We defined PLMS if PLMI $\geq 15$ [41].

\section{Statistical analysis}

Statistical analysis was carried out, using the SPSS 17.0 (SPSS Inc., Chicago, IL, USA). Data were summarized using proportions, mean \pm standard deviation, or median (interquartile range $-\mathrm{IQR}$ ), as appropriate. Data were analyzed using ANCOVA or Kruskal-Wallis test, as appropriate. Sidac post hoc analyses or Mann-Whitney test with Bonfferoni correction were conducted to explore the 
differences of the sleep parameters between moon phase groups. The significance level was defined as $\mathrm{p}<0.05$.

Tests for interaction (ANCOVA) between gender and lunar phases were used to examine whether the association between sleep parameters and moon cycle is different between men and women. We investigated the association of lunar phases and objective sleep efficiency, light sleep $\%$, deep sleep $\%$, wake\%, REM\%, sleep latency and REM latency. Skewed variables were square root- or ranktransformed, as appropriate. We adjusted our analyses for several co-variables, reportedly associated with sleep characteristics: age [42], gender [33], smoking [43], coffee consumption [44], use of sleeping pills [45], regular exercise [46], Charlson Comorbidity Index and CES-D score [47].

Moon phase was determined using information from the Hungarian Astronomical Association sidereal calendar [48]. We analyzed our data in three different ways: first, we categorized lunar phases into three groups: new moon, full moon and alternate moon (Waxing Crescent, Waxing Gibbous, Waning Gibbous and Waning Crescent). For sensitivity analysis we repeated our analysis using three different cut off points: 1) New/Full moon and one day before and one day after; 2 : New/Full moon and two days before and after; 3: New/Full moon and three days before and after.

\section{RESULTS}

Five hundred and twenty-two consecutive patients data were enrolled; two patients had a sleep efficiency under $20 \%$, four recordings were excluded due to technical limitations and 197 patients had mild/severe obstructive sleep apnea. Thus, 319 patients were included in the final analysis. 
The participant characteristics are presented in Table 1 . The mean $( \pm \mathrm{SD})$ age of the study participants was $45 \pm 14$ years for men and $52 \pm 12$ years for women. The mean BMI was $28.2 \pm 5.4 \mathrm{~kg} / \mathrm{m}^{2}$. Twenty three individuals used sleeping pills. Mean sleep efficiency was $78.2 \%$. The median [interquartile range] Charlson Comorbidity Index was 1.0 [2.0].

One hundred and seventeen patient (36\%) had mild obstructive sleep apnea, their mean AHI was 9.3 \pm 2.2 , one hundred and fourteen (35\%) had PLMS, their mean PLMI was 42.0 \pm 28.4 , while one ninety-five $(29.7 \%)$ of our patients reported insomnia symptoms, their mean Athens score was 13.5 \pm 3.5 . There were no significant differences regarding age, gender, BMI, use of sleeping pills, coffee consumption, shift work, Charlson Comorbidity Index, CESD score and sleep disorders in the study sub-groups (Table 1).

Objective sleep parameters, recorded with polysomnography, showed significant associations with lunar phases (Table 2). Deep sleep $(\mathrm{p}<0.001)$, wake $(\mathrm{p}<0.001)$ and REM $(\mathrm{p}=0.002)$ percentages were significantly different between the three groups. Post hoc analyses revealed less deep sleep percentage (6.1[4] vs. 10.9[9], $\mathrm{p}<0.05)$ and REM percentage $(10.1 \pm 6.6$ vs. $13.9 \pm 6.6, \mathrm{p}<0.05)$, higher wake percentage $(28.7 \pm 12.3$ vs. $20.2 \pm 12$, p $<0.05)$ at full moon compared to alternate moon phases. Sleep efficiency was significantly shorter $(\mathrm{p}<0.001)$, while sleep latency $(\mathrm{p}=0.001)$ and REM latency $(p<0.001)$ was longer at full moon compared to other two moon phases. The results were similar in our sensitivity analyses using the three cut off points to define "full moon" specified in the methods section (not shown).

These associations remained significant after adjusting for age, gender, smoking, coffee consumption, use of sleeping pills, and regular exercise. Most of these associations remained significant after adjustment for the Charlson Comorbidity Index. Finally, adjustment for the Center for Epidemiologic 
Studies Depression Scale score in the final model did not abrogate the significant associations for deep sleep, wake percentage, sleep efficiency, and REM latency.

The interaction between gender and lunar phases was significant for deep sleep $(\mathrm{p}=0.01)$ and sleep latency $(\mathrm{p}=0.01)$, and in general, the observed differences for the variables of interest were consistently more pronounced in women (Table 3). Interestingly, none of the assessed sleep parameters (neither subjective nor objective) were associated with pre- or postmenopausal status in this sample (not shown).

Subjective sleep-related parameters were not significantly different between the three groups (Table 4). Within gender strata, however, the self-reported sleep onset latency was longer at full moon in women (median[IQR]: new moon 30.0[25] min, full moon 52.5[66] min, alternate moon 25.0[50], $\mathrm{p}=0.04$ ), and the difference was nearly significant among men (median[IQR]: new moon 30.0[45] min, full moon 60.0[76] min, alternate moon 30.0[45] $\min , \mathrm{p}=0.06)$. 


\section{DISCUSSION}

In this cohort of patients undergoing diagnostic polysomnography, we found that full moon was associated with less deep sleep, lower sleep efficiency, and longer REM latency. Furthermore, this association seemed to be more pronounced in women than men. A novelty of our study is the enrollment of patients referred to a sleep clinic, thereby extending previous observations regarding the association between lunar cycle and sleep variables to a clinical population with potential sleep disorders.

A few earlier studies offered support for an association between the lunar cycle and human behavior [7], but others did not find such a relationship [24].

A recent study reported similar results to the ones presented here. Those authors found that around full moon deep slow-wave sleep was decreased [31]. In our analysis we also found less deep sleep at the time of full moon, compared to other lunar phases.

Two previous studies reported that that people slept on average 20 minutes less during nights with full moon compared with new moon $[27,31]$. In our analysis, we controlled for important covariates, including comorbidity and depressive symptoms, and the lunar phase remained independently associated with sleep efficiency. Similarly to our results, Cajochen et al. also reported a longer sleep latency and a decreased slow-wave sleep around full moon compared to other lunar phases [31]. They also found reduced sleep quality and total sleep time 0-4 days around the full moon. In our study, participants had been referred for a sleep center because of clinical suspicion for sleep disturbances, while the previous study enrolled healthy volunteers. Despite this relevant difference, the results are overall similar, suggesting that the observed associations might be present in subjects with sleep disorders as well as in normal sleepers [31]. 
The exact mechanism for the association between lunar cycle and sleep remains unclear, and additional studies are needed to confirm these findings and also to find potential mechanisms. Several potential mechanisms may be considered to explain these findings. When the moon is full, the Earth is between the Sun and the Moon. It cannot be excluded that the change in the electromagnetic radiation and/or the gravitational "pull off" of the moon during this phase may influence the release of neurohormones or neural activity [7]. Several observations suggest that the lunar tidal force affects certain biochemical processes [49]. A few studies have evaluated circalunar patterns of seizure occurrence [50, 51]. Furthermore, Transcranial Magnetic Stimulation (TMS) initiates EEG slowwave activity in sleeping volunteers, suggesting that electromagnetic exposures may influence brain activity and sleep characteristics [52]. Thus, the solar radiation reflected by the full moon [53], and the lunar tidal force, might also modify the brain activity.

A nocebo effect is also possible, meaning that people tended to go to bed later during full moon because they expected to sleep less well, knowing that it was full moon [27]. However, we believe that this was unlikely to influence our results since people living in large cities are less likely to be aware of lunar cycle, and the majority of the participants in fact lived in the capital of Hungary. Furthermore, neither the patients, nor the personnel were aware of our planned analyses of the lunar cycle. Finally, our sleep suits had no windows; therefore, there is little chance that expectations related to the full moon modified our results.

Association between lunar phase and sleep might be explained by circalunar periodicity of serum hormone levels. Some animals are clearly influenced by the moon and possess internal clocks that follow the lunar cycle [54-56]. In humans, circalunar hormone cycle has been observed in both women (menstrual cycle rhythmicity) and men [57]. Celec et al. found a circalunar cycle of salivary testosterone and visual-spatial performance in young healthy volunteers of both sexes [58]. Cajochen 
et al. suggested that the observed rhythm in sleep variables represents a circalunar rhythm property, running in synchrony with the lunar phases, reminiscent of other endogenous rhythms such as the circadian and circannual rhythms [31]. In addition, as mentioned before, there is a strong link between female sex hormones and sleep [32], and female reproductive steroids appear to be involved in the cardiac autonomic control during sleep in women [59]. This implies the existence of sex differences in the structure of sleep, as well as in the association between lunar phases and sleep. Although we did not measure hormone levels in our participants, therefore we cannot analyze these associations, our results suggest an increased sensitivity of women to lunar modulation.

In earlier times, before modern lighting, the light of a full moon could have kept people up at night, leading to sleep deprivation and consequent psychological issues, according to a hypothesis that awaits experimental support [60]. However, as Rotton et al. pointed out, the moon is a relatively minor source of light in our times, therefore human behavior would be much more erratic if it were affected by light levels of this magnitude [61].

Our multivariable adjusted models suggest that comorbidity and depressive symptoms are associated with sleep, as reported before [47]. The association of full moon with sleep efficiency and other sleep parameters remained significant after correcting for depression and comorbidity, and thus it is unlikely that these factors could explain our findings.

One of the strengths of our work is the relatively large sample with polysomnography data. Neither the patients, nor the personnel in the sleep laboratory were aware of our planned analysis, thus avoiding the confounding of self-fulfilling prophecy regarding a lunar influence on sleep. We adjusted for several potential confounding covariates and also analyzed the gender interaction. We 
excluded the effect of light, furthermore everyone slept under standard circumstances, bedtime and wake up was at same time for the patients.

The retrospective, cross-sectional design prevented us of detecting the temporal change of sleep charactersitics as a function of the moon cycle in a within subject design. Secondly, we enrolled individuals referred to a sleep clinic for assessment, therefore the sample was not representative of the healthy general population. Furthermore, the fact that these participants had clinical suspicion for sleep problems, might have made them more sensitive to the lunar cycle. Another limitation of this study is the absence of the normal control group and hormonal profile measurements.

If other studies confirm our findings, we believe this could potentially influence clinical practice. We do ask patients during a comprehensive sleep evaluation not only about their health but also about "environmental factors" impacting their sleep (noise, light, etc). We consider pain, disturbing bedpartners or other potential interferences, both internal and external, when assessing sleep. In the same framework, we could include the lunar effect as a potential factor. If we detect that an individual is sensitive to the effects of moon phases, this could be explained to the patient, and it could be used during the treatment. Patients could be prepared for this effect, for example in terms of sleep related expectations.

\section{CONCLUSION}

The main result of this cross-sectional study is that there is an association of moon phases with both subjective and objective sleep parameters. Full moon was associated with a significantly reduced sleep efficiency and other sleep parameters; therefore, we propose that lunar phases may contribute to the complex regulation of sleep, especially in women. Accordingly, poor sleep and the consequent emotional lability and reduced daytime functioning can be partially related to the full moon. 


\section{Acknowledgments}

The authors thank the patients and the staff of 1st Dept. of Internal Medicine, Sleep Laboratory, Semmelweis University, in Budapest, Hungary, for their assistance in this survey. Special thanks to Zsolt Turányi for his essential help in sensitivity analysis. 
References

1. Anthony, A., ed. Empires of Time: Calendars, Clocks and Cultures Social Studies. 2000, Tauris Parke Paperbacks: London. 359.

2. $\quad$ Vance, D.E., Belief in lunar effects on human behavior. Psychol Rep, 1995. 76(1): p. 32-4.

3. Wilson, J.E., 2nd and J.J. Tobacyk, Lunar phases and crisis center telephone calls. J Soc Psychol, 1990. 130(1): p. 47-51.

4. Iosif, A., Ballon, B, Bad moon rising: the persistent belief in lunar connections to madness. CMAJ, 2005(173): p. 1498-1500.

5. Cutler, W.B., Lunar and menstrual phase locking. Am J Obstet Gynecol, 1980. 137(7): p. 834-9.

6. Law, S.P., The regulation of menstrual cycle and its relationship to the moon. Acta Obstet Gynecol Scand, 1986. 65(1): p. 45-8.

7. Zimecki, M., The lunar cycle: effects on human and animal behavior and physiology. Postepy Hig Med Dosw (Online), 2006. 60: p. 1-7.

8. Ghiandoni, G., et al., Some unexpected results in time distribution analysis of spontaneous deliveries. Gynecol Obstet Invest, 1998. 46(2): p. 88-90.

9. Ghiandoni, G., et al., Incidence of lunar position in the distribution of deliveries. A statistical analysis. Minerva Ginecol, 1997. 49(3): p. 91-4.

10. Waldhoer, T., G. Haidinger, and C. Vutuc, The lunar cycle and the number of deliveries in Austria between 1970 and 1999. Gynecol Obstet Invest, 2002. 53(2): p. 88-9.

11. Morton-Pradhan, S., R.C. Bay, and D.V. Coonrod, Birth rate and its correlation with the lunar cycle and specific atmospheric conditions. Am J Obstet Gynecol, 2005. 192(6): p. 19703.

12. Arliss, J.M., E.N. Kaplan, and S.L. Galvin, The effect of the lunar cycle on frequency of births and birth complications. Am J Obstet Gynecol, 2005. 192(5): p. 1462-4.

13. Sitar, J., The causality of lunar changes on cardiovascular mortality. Cas Lek Cesk, 1990. 129(45): p. 1425-30.

14. Oomman, A., et al., A novel trigger for acute coronary syndromes: the effect of lunar cycles on the incidence and in-hospital prognosis of acute coronary syndromes--a 3-year retrospective study. J Indian Med Assoc, 2003. 101(4): p. 227-8.

15. Roman, E.M., et al., The influence of the full moon on the number of admissions related to gastrointestinal bleeding. Int J Nurs Pract, 2004. 10(6): p. 292-6.

16. Mikulecky, M. and I. Schreter, Occurrence of acute infectious diarrhea during the lunar phases. Cas Lek Cesk, 1993. 132(16): p. 498-501.

17. Zettinig, G., et al., Appointments at a thyroid outpatient clinic and the lunar cycle. Wien Klin Wochenschr, 2003. 115(9): p. 298-301.

18. Martin, S.J., I.W. Kelly, and D.H. Saklofske, Suicide and lunar cycles: a critical review over 28 years. Psychol Rep, 1992. 71(3 Pt 1): p. 787-95.

19. Mathew, V.M., et al., Attempted suicide and the lunar cycle. Psychol Rep, 1991. 68(3 Pt 1): p. 927-30.

20. Rogers, T.D., G. Masterton, and R. McGuire, Parasuicide and the lunar cycle. Psychol Med, 1991. 21(2): p. 393-7.

21. Byrnes, G. and I.W. Kelly, Crisis calls and lunar cycles: a twenty-year review. Psychol Rep, 1992. 71(3 Pt 1): p. 779-85. 
22. Benbadis, S.R., et al., The influence of the full moon on seizure frequency: myth or reality? Epilepsy Behav, 2004. 5(4): p. 596-7.

23. Neal, R.D. and M. Colledge, The effect of the full moon on general practice consultation rates. Fam Pract, 2000. 17(6): p. 472-4.

24. Foster, R.G. and T. Roenneberg, Human responses to the geophysical daily, annual and lunar cycles. Curr Biol, 2008. 18(17): p. R784-R794.

25. Alonso, Y., Geophysical variables and behavior: LXXII. Barometric pressure, lunar cycle, and traffic accidents. Percept Mot Skills, 1993. 77(2): p. 371-6.

26. Calver, L.A., B.J. Stokes, and G.K. Isbister, The dark side of the moon. Med J Aust, 2009. 191(11-12): p. 692-4.

27. Roosli, M., et al., Sleepless night, the moon is bright: longitudinal study of lunar phase and sleep. J Sleep Res, 2006. 15(2): p. 149-53.

28. Binkley, S., Wrist activity in a woman: daily, weekly, menstrual, lunar, annual cycles? Physiol Behav, 1992. 52(3): p. 411-21.

29. Binkley, S., et al., Human daily rhythms measured for one year. Physiol Behav, 1990. 48(2): p. 293-8.

30. Schredl, M., S. Fulda, and I. Reinhard, Dream recall and the full moon. Percept Mot Skills, 2006. 102(1): p. 17-8.

31. Cajochen, C., et al., Evidence that the Lunar Cycle Influences Human Sleep. Current biology : $\mathrm{CB}, 2013$.

32. Shechter, A. and D.B. Boivin, Sleep, Hormones, and Circadian Rhythms throughout the Menstrual Cycle in Healthy Women and Women with Premenstrual Dysphoric Disorder. International Journal of Endocrinology, 2010.

33. Krishnan, V. and N.A. Collop, Gender differences in sleep disorders. Curr Opin Pulm Med, 2006. 12(6): p. 383-9.

34. Soldatos CR, D.D., Paparrigopoulos TJ. , The diagnostic validity of the Athens Insomnia Scale. Psychosom Res, 2003. 55: p. 263-7.

35. Radloff, L., The CES-D scale: a self-report depression scale for research in the general population. Psychol Measurement., 1977. 1(no. 3 summer): p. 385-401.

36. Hoddes E., V.Z., H. Smythe, R. Phillips, W. C. Dement, Quantification of Sleepiness: A New Approach. Psychophysiology, 1973. 10(4): p. 431-436.

37. Akira Usui, Y.I., Ichio Obinata, Tamio Okado, Hitoshi Fukuzawa, Shigenobu Kanba Validity of sleep log compared with actigraphic sleep-wake state II. Psychiatry and Clinical Neurosciences, 1999. 53(2): p. 183-184.

38. Charlson, M.E., et al., A new method of classifying prognostic comorbidity in longitudinal studies: development and validation. J Chronic Dis, 1987. 40(5): p. 373-83.

39. Rechtschaffen A, K.A., A manual of standardized terminology, techniques, and scoring system for sleep stages of human subjects. . National Institutes of Health, 1968. Washington DC: Publication 204.

40. Sleep-related breathing disorders in adults: recommendations for syndrome definition and measurement techniques in clinical research. The Report of an American Academy of Sleep Medicine Task Force. Sleep., 1999. 22(5): p. 667-89.

41. Medicine, A.A.o.S., International classification of sleep disorders, 2 nd ed. diagnostic and coding manual, ed. A.A.o.S. Medicine. 2005, Westchester: 
42. Ohayon, M.M., et al., Meta-analysis of quantitative sleep parameters from childhood to old age in healthy individuals: developing normative sleep values across the human lifespan. Sleep., 2004. 27(7): p. 1255-73.

43. Lavie, L. and P. Lavie, Smoking interacts with sleep apnea to increase cardiovascular risk. Sleep Med., 2008. 9(3): p. 247-53. Epub 2007 May 21.

44. Hindmarch, I., et al., A naturalistic investigation of the effects of day-long consumption of tea, coffee and water on alertness, sleep onset and sleep quality. Psychopharmacology (Berl). 2000. 149(3): p. 203-16.

45. Fratello, F., et al., Can an inert sleeping pill affect sleep? Effects on polysomnographic, behavioral and subjective measures. Psychopharmacology (Berl). 2005. 181(4): p. 761-70. Epub 2005 Sep 29.

46. Youngstedt, S.D., Effects of exercise on sleep. Clin Sports Med., 2005. 24(2): p. 355-65.

47. Mendlewicz, J., Sleep disturbances: core symptoms of major depressive disorder rather than associated or comorbid disorders. World J Biol Psychiatry., 2009. 10(4): p. 269-75. doi: 10.3109/15622970802503086.

48. Mizser, A. and J. Benkő, Meteor sidereal calendar. 2006-2010, Budapest: OOK-Press Kft.

49. Papaseit, C., N. Pochon, and J. Tabony, Microtubule self-organization is gravity-dependent. Proceedings of the National Academy of Sciences, 2000. 97(15): p. 8364-8368.

50. Quigg, M., et al., Circalunar and ultralunar periodicities in women with partial seizures. Epilepsia, 2008. 49(6): p. 1081-1085.

51. Ruegg, S., et al., Association of environmental factors with the onset of status epilepticus. Epilepsy Behav, 2008. 12(1): p. 66-73.

52. Massimini, M., G. Tononi, and R. Huber, Slow waves, synaptic plasticity and information processing: insights from transcranial magnetic stimulation and high-density EEG experiments. Eur J Neurosci, 2009. 29(9): p. 1761-70.

53. N. A. Schwadron, S.S., H. E. Spence, The CRaTER Special Issue ofSpace Weather: Building the observational foundation to deduce biological effects of space radiation. Space Weather, 2013. 11(2): p. 47.

54. Palmer, J.D., The Biological Rhythms and Clocks of Intertidal Animals. Oxford University Press, 1995: p. 217

55. Naylor, E., Marine animal behaviour in relation to lunar phase. Earth Moon Planets, 2001. 85: p. 291-302.

56. Tessmar-Raible, K., Raible, F., Arboleda, E. , Another place, another timer: marine species and the rhythms of life. Bioessays, 2011. 33: p. 165-172.

57. Rensing, L.M.-G., U. Ruoff, P., Biological timing and the clock metaphor: oscillatory and hourglass mechanisms. Chronobiol Int, 2001. 18(3): p. 329-69.

58. Celec, P., et al., The circalunar cycle of salivary testosterone and the visual-spatial performance. Bratisl Lek Listy., 2002. 103(2): p. 59-69.

59. de Zambotti, M., et al., Autonomic regulation across phases of the menstrual cycle and sleep stages in women with premenstrual syndrome and healthy controls. Psychoneuroendocrinology, 2013. 38(11): p. 2618-27.

60. Raison, C.L., H.M. Klein, and M. Steckler, The moon and madness reconsidered. J Affect Disord, 1999. 53(1): p. 99-106.

61. Rotton, J. and I.W. Kelly, Much ado about the full moon: a meta-analysis of lunar-lunacy research. Psychol Bull, 1985. 97(2): p. 286-306. 
Table 1. Basic characteristics

Age (mean \pm SD)

Gender (female n (\%))

BMI (mean \pm SD) $(\mathrm{kg} / \mathrm{m} 2)$

Use of sleeping pills $\mathrm{n}(\%)$

Coffee consumption n ( \%)

Current Smoker n ( \%)

Shift worker n (\%)

Charlson Comorbidity Index (median[interquartile range])

CES-D score (median[interquartile range])

Educational level (\%)

Less than high school diploma

High school graduate

Higher education/ University

Sleep disorders

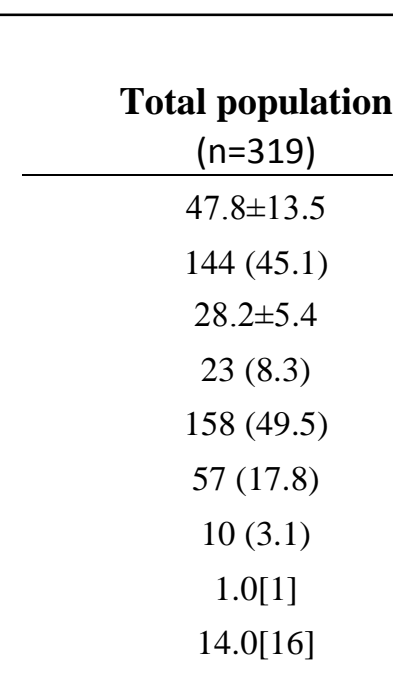

27.2

30.3

42.5

$117(9.3 \pm 2.2)$

$114(42.0 \pm 28.4)$

95(13.5 53.5$)$
PLMI $n$ (mean \pm SD)

AIS n (mean \pm SD)

\begin{tabular}{|c|c|c|}
\hline $\begin{array}{l}\text { Full Moon } \\
\qquad(n=47)\end{array}$ & $\begin{array}{l}\text { Alternate } \\
\text { Moon } \\
(n=224)\end{array}$ & p value* \\
\hline $50.4 \pm 15.8$ & $46.8 \pm 12.9$ & 0.165 \\
\hline $21(44.7)$ & $99(44.2)$ & 0.767 \\
\hline $29.7 \pm 6.6$ & $27.7 \pm 5.1$ & 0.593 \\
\hline $2(4.3)$ & $19(8.5)$ & 0.381 \\
\hline $19(40.4)$ & $124(55.3)$ & 0.917 \\
\hline $4(8.5)$ & 47 (20.9) & 0.58 \\
\hline $1(2.1)$ & $8(3.5)$ & 0.179 \\
\hline $1.0[1]$ & $1.1[1]$ & 0.280 \\
\hline \multirow[t]{2}{*}{$14.0[15]$} & $13.0[16]$ & 0.739 \\
\hline & & 0.945 \\
\hline 24.1 & 30.0 & \\
\hline 24.1 & 30.1 & \\
\hline 51.7 & 39.9 & \\
\hline $14(8.2 \pm 3.2)$ & $90(9.3 \pm 3.3)$ & 0.101 \\
\hline $15(40.1 \pm 35.1)$ & $83(44.1 \pm 28.5)$ & 0.860 \\
\hline $10(13.3 \pm 3.4)$ & $66(13.4 \pm 3.7)$ & 0.510 \\
\hline
\end{tabular}

Note: Values expressed as mean \pm SD: standard deviation, percentage (number of patients/total number of patients) or median[interquartile range],

BMI: body mass index, CES-D: Center for Epidemiologic Studies Depression Scale, AHI: apnea hypopnea index, PLMI: Periodic legs movement index,

AIS: Athens Insomnia Score; * test for heterogeneity/homogeneity Brown-Forsythe or Levene 
Table 2. Polysomnographic sleep variables

\begin{tabular}{|c|c|c|c|c|c|c|c|c|}
\hline Sleep parameter & New Moon & Full Moon & Alternate Moon & Unadjusted $\mathrm{p}$ & $\begin{array}{c}\text { Adjusted } \\
\text { model } \\
\text { p* }\end{array}$ & $\begin{array}{c}\text { Adjusted } \\
\text { model } \\
p^{* *}\end{array}$ & $\begin{array}{c}\text { Adjusted } \\
\text { model } \\
p^{* * *}\end{array}$ & Post-Hoc \\
\hline Light sleep (\%) & $56 \pm 9.9$ & $54.9 \pm 9.1$ & $54.2 \pm 10.6$ & 0.500 & 0.298 & 0.499 & 0.165 & \\
\hline Deep sleep (\%) & $8.8[9]$ & $6.1[4]$ & $10.9[9]$ & $<0.001$ & $<0.001$ & $<0.001$ & 0.002 & $\mathrm{~F}<\mathrm{A}$ \\
\hline Wake (\%) & $21.9 \pm 11.4$ & $28.7 \pm 12.3$ & $20.2 \pm 12$ & $<0.001$ & 0.004 & 0.004 & 0.024 & $F>A$ \\
\hline REM sleep (\%) & $13.1 \pm 5.8$ & $10.1 \pm 6.6$ & $13.9 \pm 6.6$ & 0.002 & 0.046 & 0.100 & 0.117 & $\mathrm{~F}<\mathrm{A}$ \\
\hline Sleep efficiency $(\%)^{\#}$ & $82.3[18]$ & $73.7[19]$ & $82.2[15]$ & $<0.001 \sim$ & 0.001 & 0.002 & 0.004 & $\mathrm{~F}<\mathrm{A}, \mathrm{F}<\mathrm{N}$ \\
\hline Sleep latency $(\mathrm{min})^{\#}$ & $12.1[14]$ & $24.5[31]$ & $13.3[19]$ & $0.001 \sim$ & 0.009 & 0.010 & 0.077 & $\mathrm{~F}>\mathrm{N}, \mathrm{F}>\mathrm{A}$ \\
\hline REM latency (min) & $98[74]$ & $137[152]$ & $97.5[76]$ & 0.001 & 0.015 & 0.034 & 0.040 & $\mathrm{~F}>\mathrm{N}, \mathrm{F}>\mathrm{A}$ \\
\hline
\end{tabular}

Notes: The results are presented as mean \pm standard deviation or median [interquartile range]. REM: Rapid eye movement, N: New Moon category, F: Full Moon category, A: Alternate moon category. ANCOVA performed on the transformed variables, but untransformed means reported for interpretability; for ANCOVAs, post-hoc tests were performed with multiple test correction according to the Sidac method. p<0.05; : KruskalWallis test, post-hoc tests were performed with Mann-Whitney tests with Bonferroni correction, $\mathrm{p}<0.0167$ ); ${ }^{\#}$ variables were rank transformed.

\footnotetext{
* age, gender, smoking, coffee use of sleeping pills, regular exercise

** also adjusted for Charlson Comorbidity Index

*** also adjusted for Center for Epidemiologic Studies Depression Scale score
} 
Table 3. Polysomnographic sleep variables by gender distribution

\begin{tabular}{|c|c|c|c|c|c|c|c|c|c|}
\hline Sleep parameter & Gender & New Moon & Full Moon & $\begin{array}{c}\text { Alternate } \\
\text { Moon }\end{array}$ & $p$ & Post-Hoc & $\begin{array}{c}\text { Adjusted } \\
\text { model } \\
\mathrm{p}^{*}\end{array}$ & $\begin{array}{c}\text { Adjusted } \\
\text { model } \\
p^{* *} \\
\end{array}$ & $\begin{array}{c}\text { Adjusted } \\
\text { model } \\
\mathrm{p}^{* * *}\end{array}$ \\
\hline \multirow[t]{2}{*}{ Light sleep (\%) } & Females & $55.6 \pm 9.1$ & $56 \pm 9.7$ & $51.9 \pm 9.3$ & 0.04 & & 0.108 & 0.315 & 0.103 \\
\hline & Males & $56.4 \pm 10.8$ & $53.8 \pm 8.9$ & $56.3 \pm 11.1$ & 0.541 & & 0.797 & 0.807 & 0.551 \\
\hline \multirow[t]{2}{*}{ Deep sleep (\%) } & Females & $6[10]$ & $6.1[3]$ & $13.5[10]$ & $<0.001$ & $N<A, F<A$ & $<0.001$ & $<0.001$ & 0.003 \\
\hline & Males & $9.6[7]$ & $6.5[4]$ & $9.4[7]$ & 0.180 & & 0.328 & 0.409 & 0.297 \\
\hline \multirow[t]{2}{*}{ Wake (\%) } & Females & $24.9 \pm 10.4$ & $30.5 \pm 10.2$ & $20.9 \pm 11.5$ & 0.001 & $F>A$ & 0.006 & 0.005 & 0.161 \\
\hline & Males & $18.8 \pm 11.8$ & $27.2 \pm 15.4$ & $19.7 \pm 12.3$ & 0.013 & $\mathrm{~F}>\mathrm{A}$ & 0.167 & 0.176 & 0.117 \\
\hline \multirow[t]{2}{*}{ REM (\%) } & Females & $11.7 \pm 5.6$ & $7.6 \pm 5.6$ & $14.12 \pm 6.4$ & $<0.001$ & $F<A$ & 0.003 & 0.012 & 0.118 \\
\hline & Males & $14.6 \pm 5.7$ & $12.1 \pm 6.7$ & $13.7 \pm 6.8$ & 0.409 & & 0.904 & 0.936 & 0.517 \\
\hline \multirow[t]{2}{*}{ Sleep efficiency $(\%)^{\#}$} & Females & $77.4[17]$ & $65.5[28]$ & $81[16]$ & $0.001^{\sim}$ & $F<A$ & 0.002 & 0.001 & 0.053 \\
\hline & Males & $84.7[19]$ & $75[18]$ & $83.1[14]$ & $0.011^{\sim}$ & $N>F, F<A$ & 0.148 & 0.165 & 0.056 \\
\hline \multirow[t]{2}{*}{ Sleep latency $(\mathrm{min})^{\#}$} & Females & $18[18]$ & $28[23]$ & $14.4[18]$ & $<0.001 \sim$ & $N<F, F>A$ & 0.012 & 0.01 & 0.363 \\
\hline & Males & $8.6[11]$ & $17.2[27]$ & $10.9[19]$ & $0.060^{\sim}$ & & 0.05 & 0.076 & 0.189 \\
\hline \multirow[t]{2}{*}{ REM latency (min) } & Females & $120.5[89]$ & $177.5[129]$ & $104[87]$ & 0.001 & $\mathrm{~N}<\mathrm{F}, \mathrm{F}>\mathrm{A}$ & 0.077 & 0.295 & 0.291 \\
\hline & Males & $81[66]$ & $122.5[141]$ & $90.2[72]$ & 0.075 & & 0.082 & 0.042 & 0.072 \\
\hline
\end{tabular}

Notes: The results are presented as mean \pm standard deviation or median [interquartile range]. N: New Moon category, F: Full Moon category, A: Alternate moon category. ANCOVA performed on the transformed variable but untransformed means reported for interpretability; for ANCOVAs, post-hoc tests were performed with multiple test correction according to the Sidac method. $\mathrm{p}<0.05$; : Kruskal-Wallis test, post-hoc tests were performed with MannWhitney tests with Bonferroni correction, $\mathrm{p}<0.0167$ ); ); ${ }^{\#}$ variables were rank transformed.

* age, gender, smoking, coffee use of sleeping pills, regular exercise

** also adjusted for Charlson Comorbidity Index

*** also adjusted for Center for Epidemiologic Studies Depression Scale score 
Table 4. Subjective sleep characteristics

\begin{tabular}{|c|c|c|c|c|}
\hline Total population & New Moon & Full Moon & $\begin{array}{c}\text { Alternate } \\
\text { Moon }\end{array}$ & $\mathrm{p}$ \\
\hline Subjective sleep onset latency (min) & $30[40]$ & $40[70]$ & $30[45]$ & 0.10 \\
\hline Subjective sleep time (h) & $6 \pm 1.7$ & $5.9 \pm 1.1$ & $5.3 \pm 1.6$ & 0.43 \\
\hline Stanford sleepiness scale & $2.6 \pm 1.1$ & $2 \pm 1.4$ & $2.5 \pm 1.1$ & 0.70 \\
\hline Morning fatigue & $3.2 \pm 1.2$ & $2.8 \pm 1.3$ & $2.6 \pm 1.2$ & 0.59 \\
\hline
\end{tabular}

Notes: The results are presented as median [interquartile range] or mean \pm standard deviation (ANOVA). 